\title{
Dispersion-optimized multicladding silicon nitride waveguides for nonlinear frequency generation from ultraviolet to mid-infrared
}

\author{
J. M. Chávez Boggio, ${ }^{1,{ }^{*}}$ A. Ortega MoñuX, ${ }^{2}$ D. Modotto, ${ }^{3}$ T. Fremberg,${ }^{1}$ \\ D. Bodenmüller, ${ }^{1}$ D. Giannone, ${ }^{1}$ M. M. Roth, ${ }^{1}$ T. Hansson, ${ }^{3,4}$ S. WABnitz, ${ }^{3}$ \\ E. Silvestre, ${ }^{5}$ AND L. ZimmermanN ${ }^{6}$ \\ ${ }^{1}$ innoFSPEC-VKS, Leibniz-Institut für Astrophysik Potsdam (AIP), An der Sternwarte 16, D-14482 Potsdam, Germany \\ ${ }^{2}$ Departamento Ingeniería de Comunicaciones, ETSI Telecomunicación, Universidad de Málaga, 29010 Málaga, Spain \\ ${ }^{3}$ Dipartimento di Ingegneria dell'Informazione, Università di Brescia, via Branze 38, 25123 Brescia, Italy \\ ${ }^{4}$ INRS-EMT, 1650 Blvd. Lionel-Boulet, Varennes, Québec J3X 1S2, Canada \\ ${ }^{5}$ Universitat de València, E-46100, Burjassot (València), Spain \\ ${ }^{6}$ IHP, Im Technologiepark 25, 15236 Frankfurt (Oder), Germany \\ *Corresponding author: jboggio@aip.de
}

\begin{abstract}
Nonlinear frequency conversion spanning from the ultraviolet to the mid-infrared (beyond $2.4 \mu \mathrm{m}$ ) is experimentally demonstrated in multicladding silicon nitride $\left(\mathrm{Si}_{X} \mathrm{~N}_{Y}\right)$ waveguides. By adjusting the waveguide cross-section the chromatic dispersion is flattened, which enhances both the efficiency and the bandwidth of the nonlinear conversion. How accurately the dispersion is tailored is assessed through chromatic dispersion measurements and an experiment/simulation comparison of the dispersive waves' wavelength locations. Undesirable fluctuations of both the refractive index and the dimensions of the waveguide during the fabrication process result in a dispersion unpredictability of at least $20 \mathrm{ps} / \mathrm{nm} / \mathrm{km}$. Finally, manipulation of the effective refractive index allows for multiple third harmonic generated tones spanning from 381 to $715 \mathrm{~nm}$.
\end{abstract}

\section{Introduction}

As light propagates in a waveguide a multitude of linear and nonlinear phenomena can occur. The ability to harness and control such phenomena is often related to the introduction of new waveguide geometries and/or the use of innovative materials with engineered optical properties. One well-known example of how new geometries enhance the efficiency of light-matter interactions came with the introduction of the photonic crystal fiber (PCF) [1]. By optimizing the hole diameter and pitch in PCFs, it is possible to tailor the chromatic dispersion and the nonlinearity while maintaining single-mode confinement, thus dramatically enhancing the efficiency of nonlinear processes [2]. As a result, broadband supercontinuum (SC) generation has, for example, become commonly accessible, triggering breakthroughs in several fields, such as metrology and spectroscopy [3,4].

In the last few years, a new frontier has opened up with the use of complementary metal-oxide semiconductor (CMOS) microelectronic technology to develop silicon nanocircuits. This approach allows the fabrication of complex photonic devices with sub- $\mu \mathrm{m}$ sizes $[5,6]$. While a wide range of nonlinear effects have been demonstrated using CMOS-compatible materials with large nonlinearity like silicon [7-9], silicon nitride [10-15], hydrogenated amorphous silicon [16], and aluminum nitride [17], the fabrication of innovative architectures with state-of-the-art precision can greatly enhance the efficiency of the nonlinear phenomena. Indeed, the ability to accurately engineer the chromatic dispersion in integrated waveguides might open new avenues for exploring a wide range of nonlinear optical effects. However, accurate dispersion control in CMOS waveguides is challenging because the fabrication process is plagued with random fluctuations that render it difficult to accurately control both the waveguide dimensions as well as its effective refractive index.

While in conventional optical fibers the dispersion can be predetermined within a precision of a few $\mathrm{ps} / \mathrm{nm} / \mathrm{km}$, in CMOS waveguides with large refractive index contrast, minute fabrication inaccuracies can potentially lead to very large dispersion fluctuations. Several numerical studies indicate that the chromatic

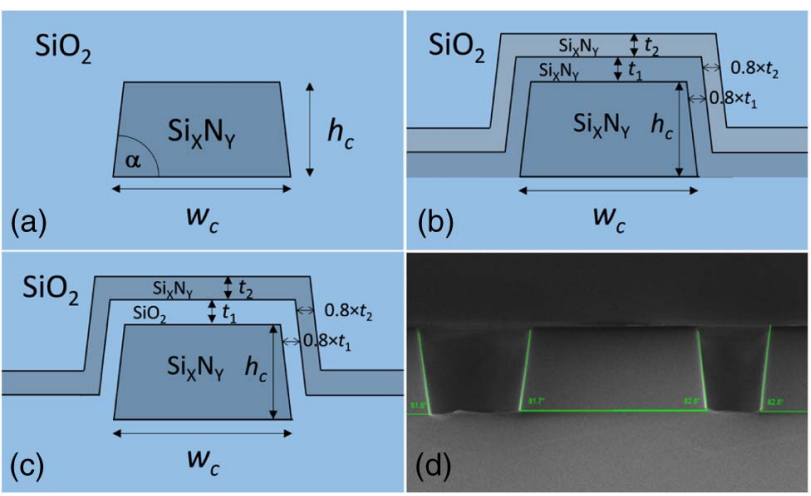

Fig. 1. Schematic of the $\mathrm{Si}_{X} \mathrm{~N}_{Y}$ waveguide cross-section. (a) Single cladding, (b) multicladding with small refractive index contrast, (c) multicladding with $\mathrm{SiO}_{2} / \mathrm{Si}_{X} \mathrm{~N}_{Y}$ refractive index contrast, and (d) SEM image of a $\mathrm{Si}_{X} \mathrm{~N}_{Y}$ etched layer.

dispersion can be engineered by the appropriate design of a multicladding cross-section [18-26]; however, a thorough experimental analysis of how accurately the dispersion can be controlled is still an issue in this technology. Furthermore, precise dispersion measurements in $\mathrm{cm}$-long waveguides are also challenging, adding to the difficulty of knowing the actual dispersion and assessing how controllable the fabrication inaccuracies can be [27].

\section{2. $\mathrm{Si}_{X} \mathbf{N}_{Y}$ Waveguides with Engineered Chromatic Dis- persion}

$\mathrm{Si}_{X} \mathrm{~N}_{Y}$ waveguides were fabricated through a plasma-enhanced chemical vapor deposition (PECVD) process, with cross-sections as shown in Figs. 1(a)-1(c) [27]. First, a thermal silica undercladding layer ( $2 \mu \mathrm{m}$ thick) is deposited on a silicon substrate. Then a $\mathrm{Si}_{X} \mathrm{~N}_{Y}$ core layer with a thickness up to $915 \mathrm{~nm}$ is added. An etching process allows the definition of core widths of $w_{c}=1.3$, 1.5 and $1.8 \mu \mathrm{m}$. Single-cladding waveguides are fabricated by depositing a 3- $\mu$ m-thick silica upper cladding on top of the $\mathrm{Si}_{X} \mathrm{~N}_{Y}$ core, as seen in Fig. 1(a). To tailor the effective refractive index 


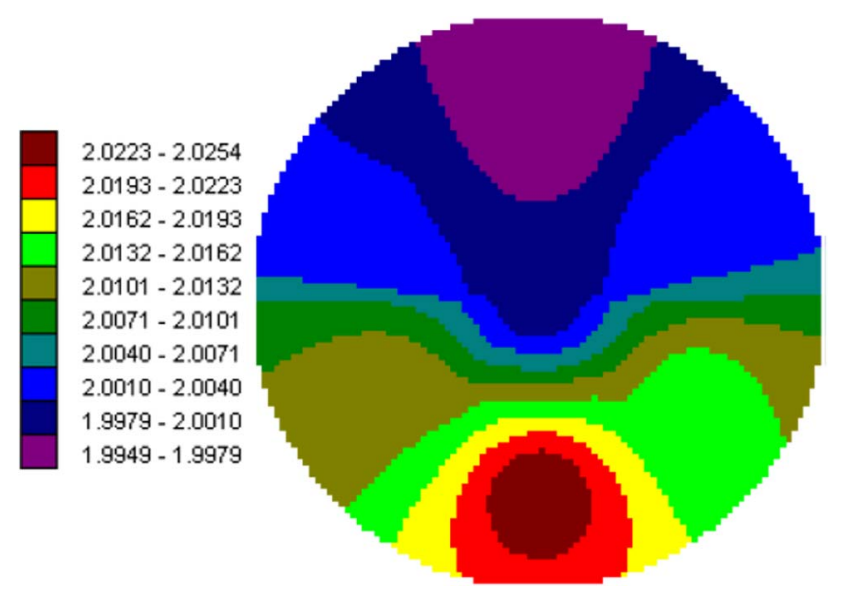

Fig. 2. Ellipsometry measurement over a $200 \mathrm{~mm}$ wafer of the $\mathrm{Si}_{X} \mathrm{~N}_{Y}$ refractive index from a film deposited using PECVD process.

in $\mathrm{Si}_{X} \mathrm{~N}_{Y}$ waveguides, two multicladding configurations are used: with either small or large refractive index contrast. For the first case, as can be seen in Fig. 1(b), on top of the $\mathrm{Si}_{X} \mathrm{~N}_{Y}$ core two additional $\mathrm{Si}_{X} \mathrm{~N}_{Y}$ layers are deposited with thicknesses up to $400 \mathrm{~nm}$ and refractive indices that are $1.5 \%$ and $3 \%$ smaller than the core, respectively. For the large contrast case depicted in Fig. 1(c), a silica cladding layer with a thickness up to $200 \mathrm{~nm}$ is deposited first, followed by a second extra cladding layer (this time made out of $\mathrm{Si}_{X} \mathrm{~N}_{Y}$ ) with the same thickness up to $t_{2}=200 \mathrm{~nm}$. Covering these two cladding layers, a 3- $\mu \mathrm{m}$ silica upper cladding is finally deposited. The waveguides exhibit a sidewall angle of $82^{\circ}$, while the sidewall thickness is $\sim 80 \%$ of the layer thickness.

A characterization of the refractive index and dimension fluctuations of core and cladding layers could not be performed once the waveguides were fabricated. Instead, to estimate those fluctuations, $\mathrm{Si}_{X} \mathrm{~N}_{Y}$ films were deposited with different thicknesses and under conditions that were similar to the actual waveguides' fabrication. Using an ellipsometry technique, the refractive index and thickness were measured over the whole $200 \mathrm{~mm}$ wafer [27]. Figure 2 shows the refractive index measurement for a $200 \mathrm{~mm}$ wafer after an annealing process was carried out to reduce losses. A peak-to-peak variation of $0.7 \%$ is measured, which will produce a dispersion uncertainty of $10 \mathrm{~s}$ of $\mathrm{ps} / \mathrm{nm} / \mathrm{km}$ [27]. While ellipsometry measurements in films estimate the parameters' fluctuations, the fabrication of waveguides with multiple cladding layers might affect those fluctuations.

\section{Experimental Procedures}

\subsection{Chromatic Dispersion Measurement}

To measure the chromatic dispersion of the $\mathrm{Si}_{X} \mathrm{~N}_{Y}$ waveguides, we use a spectral domain interferometric technique. The experimental setup is shown in Fig. 17 of [27]. The polarized light from a broadband SC source is split into two arms, one containing the $\mathrm{Si}_{X} \mathrm{~N}_{Y}$ waveguide and the other acting as the reference arm. Polarization controllers allow us to excite either the quasi-TE or the quasi-TM mode of the waveguide. The light from the two arms is then combined with a beam combiner and the interference signal is measured using two spectrum analyzers covering a spectral region from 600 to $2400 \mathrm{~nm}$. The frequency dependency of the effective refractive index accounts for a phase difference, $\phi$, between the two arms, which originates the interference spectrum.

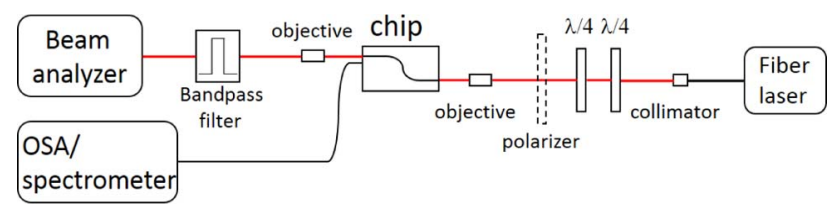

Fig. 3. Schematic of the experimental setup.

A numerical fitting of the maxima and minima of the interference signal allows us to retrieve $\frac{d \phi}{d \omega}$, which is related to the propagation constant $\beta$ through $\beta=\frac{\phi}{L}=\frac{n_{\mathrm{eff}} \omega}{c}$, where $L$ is the waveguide length, $\omega$ is the angular frequency, $n_{\mathrm{eff}}$ is the effective refractive index, and $c$ is the speed of light. The chromatic dispersion is calculated through $D=\frac{-2 \pi \beta_{2} c}{\lambda^{2}}$, where $\beta_{2}=\frac{d^{2} \beta}{d \omega^{2}}$ is the second order dispersion coefficient. The measurement was performed both with and without the chip to subtract the dispersion contribution from the splitters, combiners, objectives, etc. Since the small residual dispersion of the empty interferometer (with no chip) produces a group delay difficult to measure, it can potentially lead to a systematic error in the dispersion measurement.

\subsection{Nonlinear Frequency Generation}

Figure 3 depicts the setup used for frequency conversion in $\mathrm{Si}_{X} \mathrm{~N}_{Y}$ waveguides. The pump source is a mode-locked fiber laser, which emits pulses with a width of $85 \mathrm{fs}$ at a repetition rate of $51 \mathrm{MHz}$. The estimated peak power is $\sim 20 \mathrm{~kW}$ and the central wavelength is $\lambda_{p}=1560 \mathrm{~nm}$. We used two $\lambda / 4$ plates to collimate its light and align its polarization. A polarizer allows us to check the state of polarization (quasi-TE or quasi-TM) entering the $\mathrm{Si}_{X} \mathrm{~N}_{Y}$ waveguide. The coupling of the light into the $\mathrm{Si}_{X} \mathrm{~N}_{Y}$ waveguide is done using an objective with a 0.66 numerical aperture (NA). The losses due to the collimator, the $\lambda / 4$ plates, and the objective are measured to be $3 \mathrm{~dB}$, while the coupling losses into the waveguide are estimated to be between 6 and $8 \mathrm{~dB}$. The maximum peak power propagating in the $\mathrm{Si}_{X} \mathrm{~N}_{Y}$ waveguide is in the range $0.9-1.1 \mathrm{~kW}$ (maximum pulse energy of $110 \mathrm{pJ}$ ). The output power is collected using either a silica-lensed fiber with out-coupling losses of $6.5 \mathrm{~dB}$ or an objective with $0.7 \mathrm{NA}$ and similar losses. To visualize the spectra we use three optical spectrum analyzers (OSAs) at a 2-nm resolution covering from 350 to $2400 \mathrm{~nm}$. We check the modal characteristics of the SC and THG by using appropriate bandpass filters and a beam analyzer.

Broadband supercontinuum is best achieved when a femtosecond pump pulse propagates in the anomalous dispersion regime so that soliton-related effects dominate the broadening [4,28-31]. In the initial stages, the injected high-order soliton is subject to a strong temporal compression and the spectral broadening is triggered by the fission of the high-order soliton into fundamental soliton constituents. The soliton order is defined by $N=\sqrt{\frac{\gamma P_{0} T_{0}^{2}}{\left|\beta_{2}\right|}}$, where $\gamma=\frac{2 \pi n_{2}}{\lambda_{p} A_{\text {eff }}}$ is the nonlinear coefficient of the waveguide, $P_{0}$ is the pump peak power, $T_{0}$ is the pulse width defined as $1 / e$ halfwidth, $\lambda_{p}$ is the pump wavelength, $n_{2}$ is the nonlinear refractive index, and $A_{\text {eff }}$ is the effective area of the waveguide [31]. The soliton fission characteristic length has been empirically defined as $L_{\mathrm{fiss}} \sim \frac{L_{D}}{N}$ [31], where $L_{D}$ is the dispersion length, which is defined by $L_{D}=\frac{T_{0}^{2}}{\left|\beta_{2}\right|}$. The highest soliton pulse compression is achieved at the soliton fission length. During the fission process excess energy is radiated into dispersive waves (DWs). Depending 


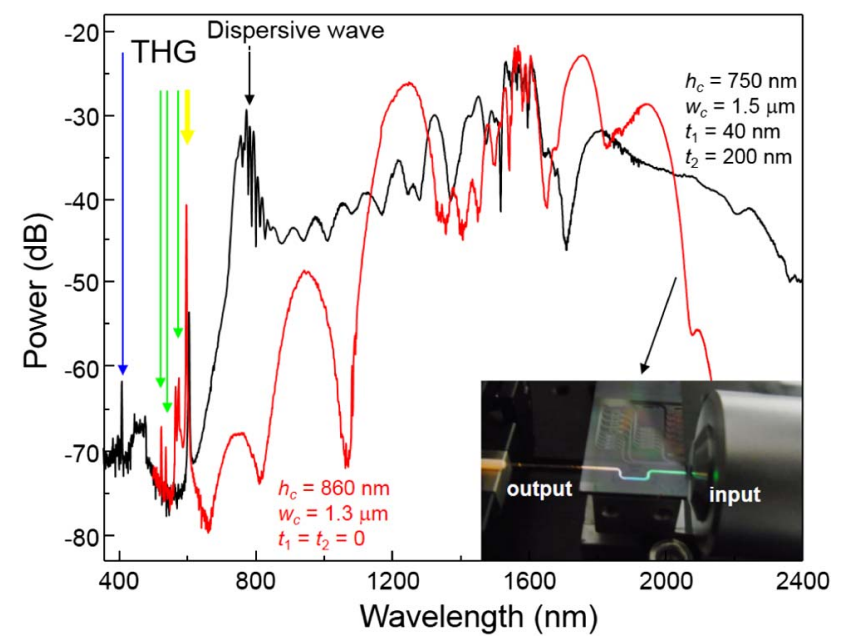

Fig. 4. $\mathrm{Si}_{X} \mathrm{~N}_{Y}$ waveguides allow for broadband $\mathrm{SC}$ and efficient third harmonic generation (THG). The flatter SC is obtained with a multicladding waveguide with $\mathrm{Si}_{X} \mathrm{~N}_{Y} / \mathrm{SiO}_{2}$ refractive index contrast, while the other spectrum is obtained with a single-cladding waveguide. Inset: scattered light shows intense THG.

on the flatness of the dispersion profile, the DWs can merge with the spectral components building up from other nonlinear effects, resulting in the production of a fairly flat SC spectrum; otherwise, the DWs emerge rather as isolated spectral bands.

Figure 4 shows two typical spectra obtained after propagating these pulses in the $\mathrm{Si}_{X} \mathrm{~N}_{Y}$ waveguides. SC light, together with multiple third harmonic tones, is generated from 410 to beyond $2400 \mathrm{~nm}$. The process of dispersive wave generation is also very efficient. The frequency location, $\omega_{\mathrm{DW}}$, of the dispersive waves is given by the phase-matching condition between the femtosecond soliton pump at frequency $\omega_{p}$ and a continuous wave

$$
\Delta \kappa=\beta\left(\omega_{\mathrm{DW}}\right)-\beta\left(\omega_{p}\right)-\left(\omega_{\mathrm{DW}}-\omega_{p}\right) / u_{g}-\gamma P_{0}=0,
$$

where $\beta$ is the propagation constant evaluated at frequencies $\omega_{\mathrm{DW}}$ and $\omega_{p}$, and $u_{g}$ is the group velocity at the pump frequency $\omega_{p}[29,30]$. We must emphasize that both the spectral position and the amplitude of the DWs are very sensitive to the dispersion profile [32].

In addition to the $\mathrm{SC}$ broadening, an extra enhancement of the frequency conversion bandwidth is obtained through third harmonic generation (THG) [13,33-35], whose efficiency is maximized when the following phase-matching condition is satisfied: $n_{\text {eff }}^{1}(\omega)=$ $n_{\text {eff }}^{k}(3 \omega)$, where $n_{\text {eff }}^{1}(\omega)\left(n_{\text {eff }}^{k}(3 \omega)\right)$ is the effective refractive index of the fundamental ( $k$ th higher order) mode at frequency $\omega(3 \omega)$.

\subsection{Measurement versus Simulation Comparison}

To efficiently perform nonlinear frequency conversion over broad bandwidths, a prerequisite is to have a proper characterization of the waveguides' chromatic dispersion in order to be able to fabricate waveguides with a pre-defined dispersion profile. This means assessing the main error sources in the dispersion engineering process. Those errors come either from the dispersion measurement itself or from a lack of precise control of the waveguide parameters (dimensions and refractive indices) due to fluctuations during the fabrication process. For this purpose, we use a full vectorial mode solver (FemSim package from RSoft) [27] to simulate the waveguide dispersion, which is compared with the measurement. If

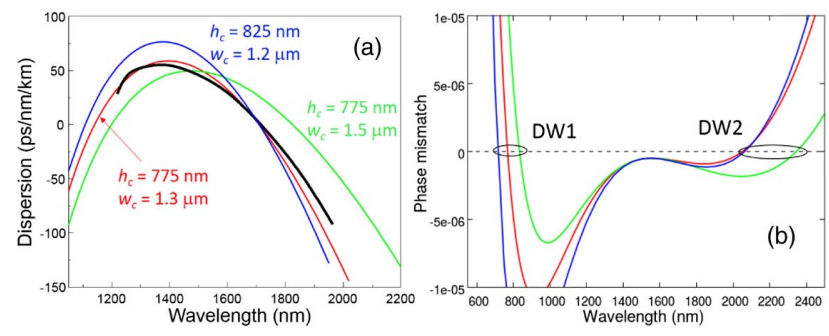

Fig. 5. (a) Measured dispersion (black line); simulation with nominal dimensions $h_{c}=775 \mathrm{~nm}$ and $w_{c}=1.5 \mu \mathrm{m}$ (green line); with $h_{c}=$ $775 \mathrm{~nm}$ and $w_{c}=1.3 \mu \mathrm{m}$ (red line); with $h_{c}=825 \mathrm{~nm}$ and $w_{c}=1.2 \mu \mathrm{m}$ (blue line). (b) Phase-mismatch, $\Delta k$, for dispersive wave generation with the dispersion profiles in (a).

using the nominal fabrication dimensions and an average refractive index value from our ellipsometry measurements does not produce good agreement between the experimental and the simulated dispersion, then the waveguide dimensions are adjusted until a good fitting is found. Using the generated dispersion curves, nonlinear simulations of the SC spectrum and the calculation of the DW locations are performed. The mismatch between the measured SC (and DW) spectrum and the numerically calculated ones tells us how accurate the dispersion measurement itself is and the uncertainty of the dimension and refractive index of the waveguide related to fabrication fluctuations.

Even though in our waveguides up to four modes can be excited at $1560 \mathrm{~nm}$ [27], we assume in our analysis that most of the power propagates in the fundamental mode. This assumption is corroborated by simulations that show that essentially only the fundamental mode is excited by centered-injection of the pump light into the waveguides.

\section{Single-Cladding $\mathrm{Si}_{X} \mathrm{~N}_{Y}$ Waveguide}

Figure 5(a) depicts with a black line the measured chromatic dispersion of a single-cladding waveguide [Fig. 1(a)] having nominal dimensions $h_{c}=775 \mathrm{~nm}$ and $w_{c}=1.5 \mu \mathrm{m}$ and a length $L=4.84 \mathrm{~cm}$. The measurement corresponds to the excitation of the quasi-TE mode. For comparison, numerical calculations of the chromatic dispersion are done using the nominal dimensions of $h_{c}=775 \mathrm{~nm}$ and $w_{c}=1.5 \mu \mathrm{m}$ and a sidewall angle of $82^{\circ}$. The refractive index in the simulation is the average value from our ellipsometry measurements. The calculated dispersion, depicted as a green solid line in Fig. 5(a), shows a non-negligible disagreement with the measured dispersion. However, the red solid line, which is obtained with $h_{c}=775 \mathrm{~nm}$ and $w_{c}=1.3 \mu \mathrm{m}$, shows a very good accord to the dispersion measurement. While the fabrication inaccuracy (within three standard deviations) of the core width $w_{c}$ is expected to be $0.15 \mu \mathrm{m}$, the value of $w_{c}$ necessary to have a good accord with the measurement is slightly outside this expected fluctuation range. Therefore, to better assess the accuracy of the dispersion measurement itself, it is necessary to resort to nonlinear measurements and simulations. Figure 5(b) shows the calculated phase mismatch for the generation of dispersive waves using Eq. (1) and the dispersion profiles plotted in Fig. 5(a). The dispersion profiles in green and red produce phase-matching wavelengths for the generation of the DW1 that are separated by $80 \mathrm{~nm}$, while for the DW2 they are $250 \mathrm{~nm}$ apart. This spectral variation of the DW location can easily be measured in experimental SC spectra to check for the more accurate dispersion profile. 

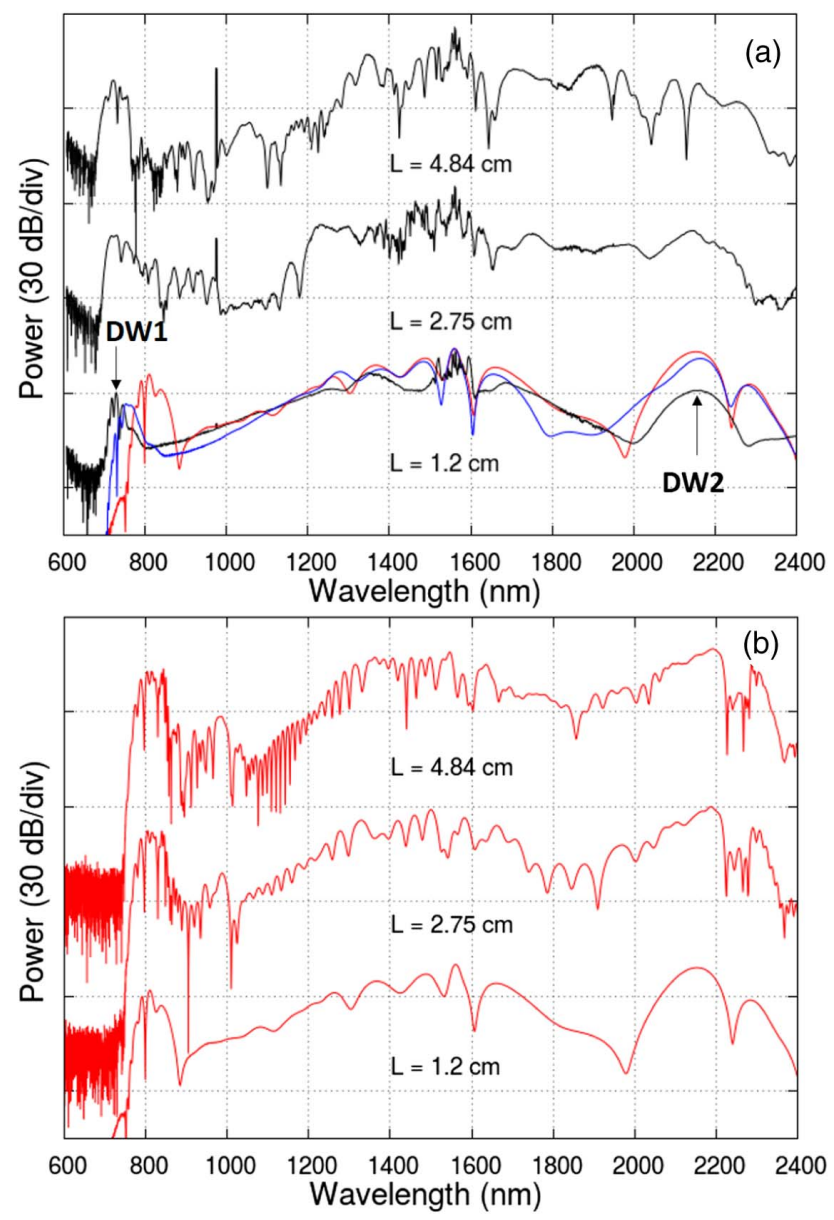

Fig. 6. SC generation. (a) Experiment (black line); simulation (red line) obtained with dispersion in Fig. 4(a) $\left(h_{c}=775 \mathrm{~nm}\right.$ and $\left.w_{c}=1.3 \mu \mathrm{m}\right)$; simulation (blue line) obtained with dispersion in Fig. $4\left(\right.$ a) $\left(h_{c}=825 \mathrm{~nm}\right.$ and $w_{c}=1.2 \mu \mathrm{m}$ ). (b) Numerical simulations.

Light from the femtosecond laser with $P_{0} \approx 500 \mathrm{~W}$ is injected into the quasi-TE mode of the waveguide characterized in Fig. 5(a). The measured SC spectra for $L=1.2,2.75$, and $4.84 \mathrm{~cm}$ are shown by the black lines in Fig. 6(a). The SC spans roughly from 700 to $2400 \mathrm{~nm}$ and the generation of two DWs is clearly noted at 740 and $2170 \mathrm{~nm}$. For our parameters $\left(\gamma \approx 1 /(\mathrm{W}-\mathrm{m}), P_{0} \approx 500 \mathrm{~W}\right.$, $T_{0}=85 \mathrm{fs}$, and $D \approx 35 \mathrm{ps} / \mathrm{km} / \mathrm{nm}$ ) the soliton number is $N \approx 8.5$, while the characteristic soliton fission length is $L_{\mathrm{fiss}} \approx 1.7 \mathrm{~cm}$. Therefore, soliton fission is the dominant mechanism for the SC dynamics along most of the propagation length. Note that, while the SC spectrum for the $\mathrm{Si}_{X} \mathrm{~N}_{Y}$ waveguide with $L=1.2 \mathrm{~cm}$ is smooth, for longer propagation lengths $(L=2.4 \mathrm{~cm}$ and $L=4.84 \mathrm{~cm})$ the SC spectra contains several dips. This result is due to the interference of the various soliton components, traveling at different group velocities, coming from the fission of the injected higherorder soliton. The occurrence of the structured SC spectrum at $L=2.4 \mathrm{~cm}$ and $L=4.84 \mathrm{~cm}$ (but not at $L=1.2 \mathrm{~cm}$ ) is in agreement with the calculated $L_{\text {fiss }} \approx 1.7 \mathrm{~cm}$.

To simulate the propagation of femtosecond pulses in the $\mathrm{Si}_{X} \mathrm{~N}_{Y}$ waveguides, a generalized nonlinear Schrödinger Equation (NLSE) is employed [31]. The NLSE includes the Kerr nonlinearity, self-steepening, attenuation, as well as all orders of chromatic dispersion using dispersion profiles obtained from experiments or RSoft simulations. Raman scattering is not included due to its narrow gain bandwidth in $\mathrm{Si}_{X} \mathrm{~N}_{Y}$. For the results in Fig. 6(b),
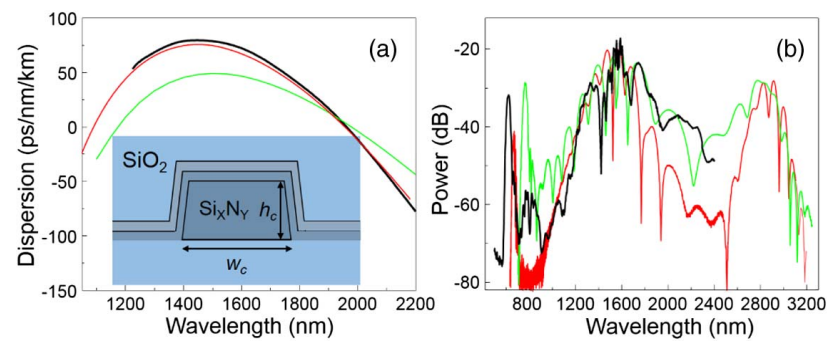

Fig. 7. (a) Measured dispersion (black line). Simulation with nominal dimensions (green line); with $h_{c}=890 \mathrm{~nm}, w_{c}=1.45 \mu \mathrm{m}$, and $t_{1}=t_{2}=40 \mathrm{~nm}$ (red line). (b) Measured SC generation (black line). Simulated SC with green (red) dispersion profile [green (red) line].

the dispersion input was the curve plotted with the solid red line in Fig. 5(a). The transition between the smooth $(L=1.2 \mathrm{~cm})$ and the structured $(L=2.4$ and $4.84 \mathrm{~cm}) \mathrm{SC}$ spectrum is clearly observed in the simulations, which is in good agreement with the experimental results. Figure 6(a) includes a red curve that shows the simulated SC spectrum, which is calculated with the dispersion profile plotted as a red solid line in Fig. 5(a). The agreement is quite remarkable except the location of the DW1: In the experiment, it is at $740 \mathrm{~nm}$, while in the simulation it is at $800 \mathrm{~nm}$. By adjusting the waveguide dimensions to $h_{c}=825 \mathrm{~nm}$ and $w_{c}=1.2 \mu \mathrm{m}$ we obtained the dispersion profile in blue in Fig. 5(a) that generates the SC spectrum in blue in Fig. 6(a). A better agreement between the experimental and the simulated DW1 location is found with this dispersion profile.

\section{Multicladding Waveguide with Small Refractive In- dex Contrast}

The black line in Fig. 7(a) shows the measured dispersion for the quasi-TE mode of a waveguide with a refractive index arrangement as depicted in Fig. 1(b) and nominal dimensions $h_{c}=790 \mathrm{~nm}$, $w_{c}=1.5 \mu \mathrm{m}$, and $t_{1}=t_{2}=100 \mathrm{~nm}$. The dispersion flatness is not much different with respect to the single cladding case in Fig. 5(a). For comparison, the solid green line shows the dispersion calculated using the nominal fabrication parameters. The measured dispersion profile has the same shape as the calculated one, but it is vertically up-shifted by $\sim 40 \mathrm{ps} / \mathrm{nm} / \mathrm{km}$. A good agreement, as shown with the red dispersion profile, can only be obtained with dimensions that are outside the expected range of fabrication fluctuations: $h_{c}=890 \mathrm{~nm}, w_{c}=1.45 \mu \mathrm{m}$, and $t_{1}=t_{2}=40 \mathrm{~nm}$. This indicates that the discrepancy between experiment and simulations comes not only from dimension uncertainties but also from the $\mathrm{Si}_{X} \mathrm{~N}_{Y}$ refractive index fluctuations during the various fabrication steps.

The black solid line in Fig. 7(b) shows the measured SC spectrum for this waveguide $(L=1.2 \mathrm{~cm})$ after exciting the quasi-TE mode with $P_{0} \approx 500 \mathrm{~W}$. Since the dispersion at the pump wavelength is large $(75 \mathrm{ps} / \mathrm{nm} / \mathrm{km})$, the generated SC is not as flat as in the case of Fig. 6, which originates from a lower dispersion $(D \approx 35 \mathrm{ps} / \mathrm{nm} / \mathrm{km})$. The green line shows the calculated SC spectrum using the chromatic dispersion depicted in green in Fig. 7(a). It presents a strong DW1 centered at $770 \mathrm{~nm}$; in the experiment it is at $630 \mathrm{~nm}$. This discrepancy is the footprint of the uncertainties in retrieving the actual dispersion profile. The SC simulation in red was obtained using the dispersion profile that matched the measured dispersion [plotted in red in Fig. 7(a)]. It produces a DW1 at $670 \mathrm{~nm}$ that is in better agreement with the 

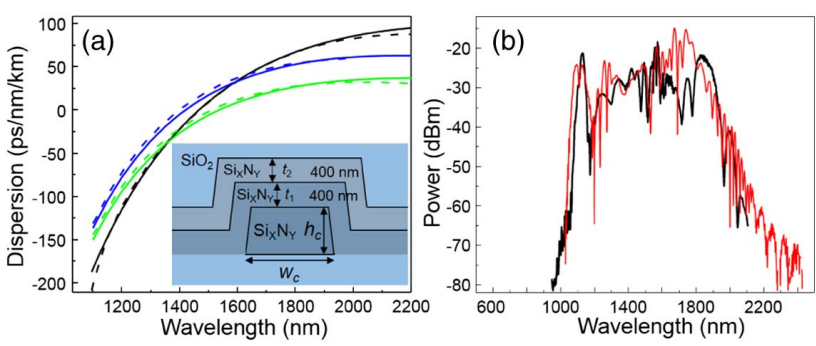

Fig. 8. (a) Measured dispersion (black line); simulation (green lines, nominal parameters); $h_{c}=860 \mathrm{~nm}$ and $t_{1}=t 2=350 \mathrm{~nm}$ (blue lines). For all results, $w_{c}=1.8 \mu \mathrm{m}$ (dashed) and $w_{c}=1.3 \mu \mathrm{m}$ (solid). (b) Measured SC generation (black line). Simulated SC using the measured dispersion profile (red line).

experiment, which indicates that the dispersion measurement is accurate.

Figure 8(a) shows the dispersion measurements for the quasi-TE mode of a waveguide having nominal dimensions of $h_{c}=790 \mathrm{~nm}$, $t_{1}=t_{2}=400 \mathrm{~nm}$, and $w_{c}=1.3$ (black solid line) and $1.8 \mu \mathrm{m}$ (black dashed line). This waveguide has a single zero-dispersion wavelength and the region where the dispersion is anomalous starts at $1450 \mathrm{~nm}$ and reaches the mid infrared. For comparison, the green lines show the calculated dispersion profiles using the nominal fabrication parameters (solid line for $w_{c}=1.3 \mu \mathrm{m}$ and dashed line for $\left.w_{c}=1.8 \mu \mathrm{m}\right)$. There is a very good qualitative agreement of the dispersion shape (there is a single zero dispersion wavelength) with the measurements, but a strong quantitative disagreement on the dispersion values. By changing the parameters to $h_{c}=860 \mathrm{~nm}$ and $t_{1}=t_{2}=350 \mathrm{~nm}$ (solid line for $w_{c}=1.3 \mu \mathrm{m}$ and dashed line for $w_{c}=1.8 \mu \mathrm{m}$ ), the results shown by blue lines indicate a slightly improvement in the agreement with the measurement; however, it was not possible to have a good accord (as in the case of Fig. 5) with dimensions within the range of expected fluctuation values.

We believe that this shows, as in the case of Fig. 7, that the average refractive index value used in our simulations is the source of disagreement between the experiments and simulations. The actual $\mathrm{Si}_{X} \mathrm{~N}_{Y}$ refractive index is unknown after the several fabrication steps in our PECVD process. Figure 8(b) shows the measured SC spectrum (black solid line) in the waveguide with $w_{c}=1.8 \mu \mathrm{m}$ and $L=4.84 \mathrm{~cm}$, obtained by injecting $P_{0} \approx 500 \mathrm{~W}$ and exciting the quasi-TE mode. The red line depicts the calculated SC generated using the measured dispersion profile, which shows very good agreement with the experiment. The reason for this good agreement is because the SC bandwidth is entirely located within the region where the dispersion is measured, showing that the dispersion measurement is accurate.

\section{Multicladding Waveguide with $\mathrm{Si}_{X} \mathrm{~N}_{Y} / \mathrm{SiO}_{2}$ Refractive Index Contrast}

Multicladding waveguides with $\mathrm{Si}_{X} \mathrm{~N}_{Y} / \mathrm{SiO}_{2}$ refractive index contrast [as in Fig. 1(c)] can produce, at least in theory, very flat dispersion profiles [27]. Four chips containing multicladding waveguides were fabricated having the nominal parameters described in Table 1. From chip $A$ to $C$ only the thickness $t_{1}$ is changed, while for chip $D$ a different set of parameters was fabricated in order to render the chromatic dispersion flatter. In those chips, dispersion measurements were performed in waveguides of $L=4.85 \mathrm{~cm}$, while for the generation of SC spectra, waveguides with $L=1.4 \mathrm{~cm}$ were used.
Table 1. Transversal Dimensions of $\mathrm{Si}_{X} \mathrm{~N}_{Y}$ Waveguides

\begin{tabular}{ccccc}
\hline Chip & $h_{c}(\mathrm{~nm})$ & $w_{c}(\mu \mathrm{m})$ & $t_{1}(\mathrm{~nm})$ & $t_{2}(\mathrm{~nm})$ \\
\hline$A$ & 860 & $1.3 ; 1.5 ; 1.8$ & 60 & 120 \\
$B$ & 860 & $1.3 ; 1.5 ; 1.8$ & 90 & 120 \\
$C$ & 860 & $1.3 ; 1.5 ; 1.8$ & 120 & 120 \\
$D$ & 750 & $1.3 ; 1.5 ; 1.8$ & 40 & 200
\end{tabular}

The chromatic dispersion was measured for chips $A, B$, and $C$ and the results are plotted in Fig. 9. For the three chips, the measurements show that the dispersion profile is flattened as the core width is increased. Note also that as $w_{c}$ is increased, the location of maximum dispersion is shifted toward longer wavelengths. More importantly, from chip $A$ through chip $C t_{1}$ is changed from 60 to 90 and to $120 \mathrm{~nm}$ while the other parameters are kept constant. As shown in [27], a change of $t_{1}$ produces a vertical shift of the dispersion profile with minor changes on its shape. A comparison between Figs. 9(a) and 9(c) shows that in the former case the maximum dispersion value is $75 \mathrm{ps} / \mathrm{nm} / \mathrm{km}$, while in the latter one it is around $55 \mathrm{ps} / \mathrm{nm} / \mathrm{km}$, which is in agreement with the expected behavior with a change in $t_{1}$.

To assess the accuracy of the dispersion measurements, we resort again to comparisons both with dispersion simulations and with nonlinear experiments. We used a waveguide from chip $C$ for the quasi-TE polarization in our analysis. Figure 10(a) shows with a black solid line the measured dispersion for a waveguide with nominal dimensions $h_{c}=860 \mathrm{~nm}, w_{c}=1.3 \mu \mathrm{m}$, and $t_{1}=$ $t_{2}=120 \mathrm{~nm}$. The green curve shows the simulated dispersion using the nominal dimensions. If compared with the measured profile, the simulated one is shifted toward longer wavelengths. We found a very good accord between experiment and simulation using $h_{c}=840 \mathrm{~nm}, w_{c}=1.25 \mu \mathrm{m}$, and $t_{1}=t_{2}=60 \mathrm{~nm}$, which is plotted with a red solid line. However, the value of $t_{1}=t_{2}=60 \mathrm{~nm}$ to reach this good accord is largely outside the fluctuation margins from fabrication.

Figure 10(b) shows with a black solid line the measured SC spectrum obtained by propagating the femtosecond pump with a peak power of $P_{0} \approx 500 \mathrm{~W}$. The SC spectrum covers a range from $615 \mathrm{~nm}$ to beyond $2400 \mathrm{~nm}$. The SC flatness in this case is comparable with the SC in Fig. 6 obtained with a single-cladding waveguide, but the measured bandwidth is slightly larger, showing that multicladding waveguides with large refractive index contrast outperform the other types of geometries. The green and red plots show the simulated results obtained with the green and red simulated dispersion profiles in Fig. 10(a). There is a disagreement in the location of the DW1 for both simulated spectra; however, the red one shows better agreement, indicating that the measured dispersion is more accurate than the dispersion simulated starting from the nominal geometrical parameters.

Even though the chromatic dispersion in chips $A, B$, and $C$ is flatter than in the single-cladding waveguide (especially for $w_{c}=$ $1.8 \mu \mathrm{m}$ ), the generation of broadband and flat SC is rather difficult because at the pump wavelength $(1560 \mathrm{~nm}) D>45 \mathrm{ps} / \mathrm{nm} / \mathrm{km}$. To improve the SC bandwidth and its flatness, the dispersion must be decreased to lower values while keeping it in the anomalous regime over broad bandwidths. As shown in [27], while changing $t_{1}$ or $h_{c}$ produces a downshift of the dispersion profile with a small change on its shape, a bigger shift occurs by modifying $h_{c}$. 

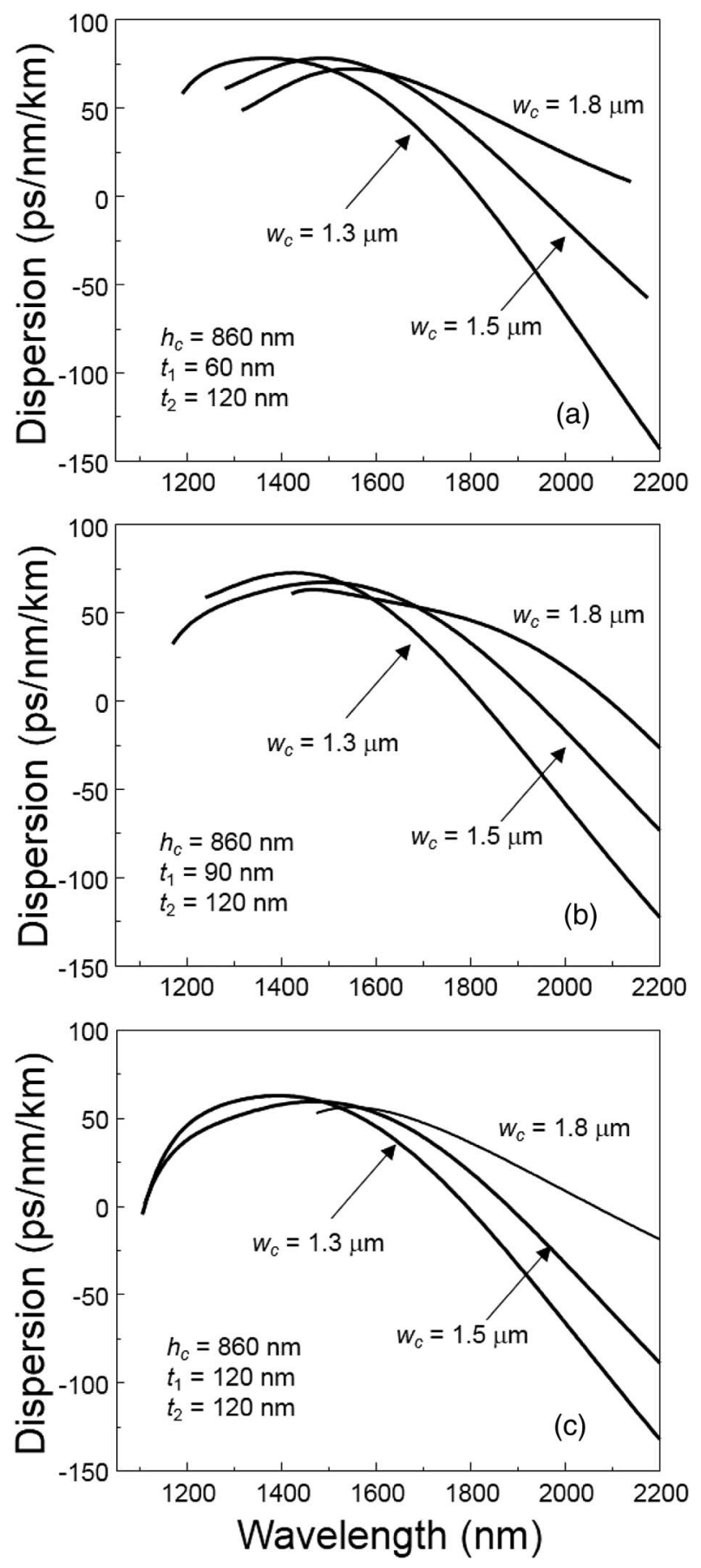

Fig. 9. Measured dispersion profiles for the quasi-TE mode. (a) Chip A, (b) chip $B$, and (c) chip $C$.

We fabricated a chip $D$ with $h_{c}=750 \mathrm{~nm}, t_{1}=40 \mathrm{~nm}$, and $t_{2}=200 \mathrm{~nm}$ and core widths of $w_{c}=1.3,1.5$, and $1.8 \mu \mathrm{m}$. The chromatic dispersion was measured for the quasi-TE and quasi-TM modes and the results are depicted in Figs. 11(a) and 11(b), respectively. The main difference between the quasi-TE and quasi-TM dispersion profiles for these dimensions is that the quasi-TE profiles are flatter. Furthermore, as in chips $A, B$, and $C$, the dispersion profile becomes progressively flatter as $w_{c}$ is increased, especially at longer wavelengths where the zero dispersion wavelength is shifted from $1600 \mathrm{~nm}$ (for $w_{c}=1.3 \mu \mathrm{m}$ ) to $1700 \mathrm{~nm}$ (for $w_{c}=1.8 \mu \mathrm{m}$ ) for the quasi-TM case and from $1770 \mathrm{~nm}$ (for $\left.w_{c}=1.3 \mu \mathrm{m}\right)$ to $2030 \mathrm{~nm}\left(w_{c}=1.8 \mu \mathrm{m}\right)$ for the quasiTE mode. The dispersion profile of the quasi-TE mode with $w_{c}=$
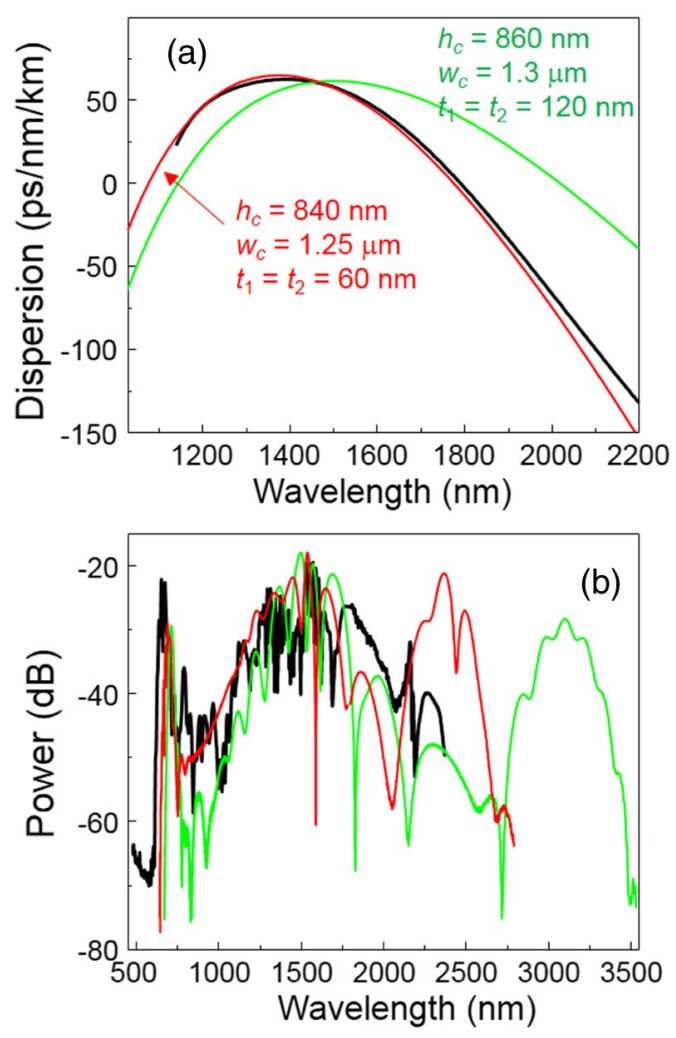

Fig. 10. (a) Measured dispersion (black line); simulation (green line, parameters); $h_{c}=840 \mathrm{~nm}, w_{c}=1.25 \mu \mathrm{m}, t_{1}=t_{2}=60 \mathrm{~nm}$ (red line). (b) Measured SC generation (black line). SC calculated [green (red) line] with green (red) simulated dispersion in Fig. 9(a).

$1.8 \mu \mathrm{m}$ has low and anomalous dispersion over almost a $1000 \mathrm{~nm}$ bandwidth. The much flatter dispersion shape in this waveguide, if compared with the ones that can be obtained in waveguides with single cladding and multicladding with small refractive index contrast, demonstrates the advantage of the $\mathrm{Si}_{X} \mathrm{~N}_{Y} / \mathrm{SiO}_{2}$ refractive index contrast profile.

The accuracy assessment of the dispersion measurements is performed for the TM case for two values of the core width. The black solid line in Fig. 12(a) shows the measured dispersion result for the waveguide with nominal dimensions $h_{c}=750 \mathrm{~nm}$, $w_{c}=1.3 \mu \mathrm{m}, t_{1}=40 \mathrm{~nm}$, and $t_{2}=200 \mathrm{~nm}$. The simulation with the nominal values is depicted in green and cannot reproduce the measured dispersion profile, but it is shifted by $220 \mathrm{~nm}$ toward longer wavelengths. We obtained a better accord with the measurement using the dimensions $h_{c}=750 \mathrm{~nm}, w_{c}=1.3 \mu \mathrm{m}$, $t_{1}=40 \mathrm{~nm}$, and $t_{2}=70 \mathrm{~nm}$, as shown in the red plot. We obtained this good accord just by modifying $t_{2}$ from its nominal value of $200 \mathrm{~nm}$ to $70 \mathrm{~nm}$. However, a value of $t_{2}=70 \mathrm{~nm}$ is outside the fabrication fluctuation margins. On the other hand, the black solid line in Fig. 12(b) shows the measured dispersion for the waveguide with nominal dimensions $h_{c}=750 \mathrm{~nm}, w_{c}=1.8 \mu \mathrm{m}, t_{1}=40 \mathrm{~nm}$, and $t_{2}=200 \mathrm{~nm}$. The green line shows the simulation using the nominal fabrication dimensions, producing a dispersion profile that is shifted by $200 \mathrm{~nm}$ toward longer wavelengths. We found a good accord with the measured dispersion simulating with the dimensions $h_{c}=750 \mathrm{~nm}, w_{c}=1.8 \mu \mathrm{m}$, and $t_{1}=40 \mathrm{~nm}$, and again with $t_{2}=70 \mathrm{~nm}$.

We gained further information about the accuracy of the dispersion measurements through nonlinear experiments. The fem- 

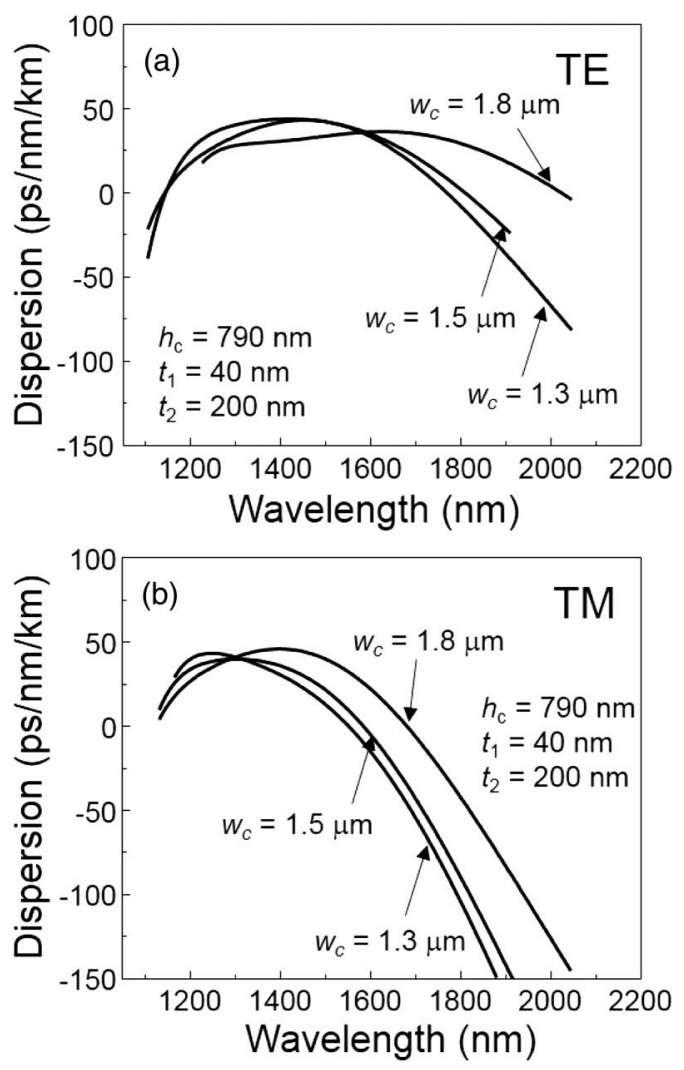

Fig. 11. Measured dispersion. (a) Quasi-TE and (b) quasi-TM. Nominal dimensions are $h_{c}=750 \mathrm{~nm}, t_{1}=40 \mathrm{~nm}$, and $t_{2}=200 \mathrm{~nm}$, and three core widths $w_{c}=1.3,1.5$, and $1.8 \mu \mathrm{m}$.
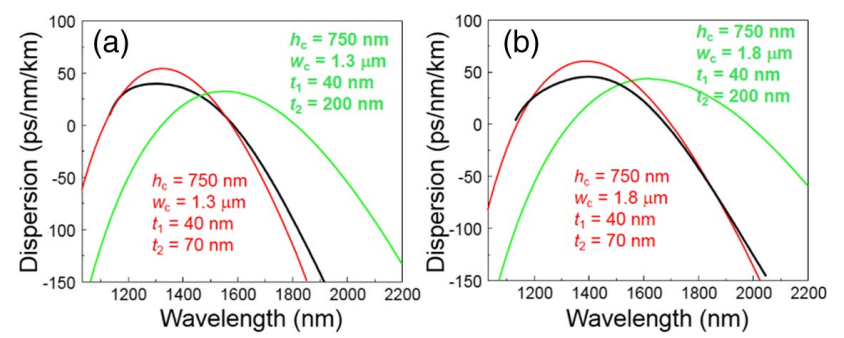

Fig. 12. Measured dispersion. (a) Quasi-TE and (b) quasi-TM. Nominal dimensions are $h_{c}=750 \mathrm{~nm}, t_{1}=40 \mathrm{~nm}$, and $t_{2}=200 \mathrm{~nm}$, and three core widths $w_{c}=1.3,1.5$, and $1.8 \mu \mathrm{m}$.

tosecond pump was propagated with a peak power of $P_{0} \approx 900 \mathrm{~W}$ through the waveguides in chip $D$ with core width $1.8 \mu \mathrm{m}$. Figure 13 shows the SC spectra for the $\mathrm{Si}_{X} \mathrm{~N}_{Y}$ waveguide having $w_{c}=1.8 \mu \mathrm{m}$ for both the quasi-TE mode and the quasi-TM mode propagation. Note that the $\mathrm{Si}_{X} \mathrm{~N}_{Y}$ waveguide with quasi-TE mode has a flatter SC spectrum and it extends beyond $2400 \mathrm{~nm}$ while the SC in the quasi-TM case abruptly ends at $2200 \mathrm{~nm}$. This is explained by the fact that the quasi-TM dispersion profile has a zero dispersion wavelength at $1700 \mathrm{~nm}$ but for the quasi-TE profile it is at $2030 \mathrm{~nm}$, which makes the SC extend over a broader bandwidth. To assess the accuracy of the dispersion measurements, we performed the SC simulations using profiles that fit the measured dispersion profiles.

The agreement between the experimental and calculated SC is good for both the quasi-TM and quasi-TE cases. The quasi-TM SC simulation exhibits a DW1 at $810 \mathrm{~nm}$, while in the measurement it is at $770 \mathrm{~nm}$, and at long wavelengths, the simulated SC shows
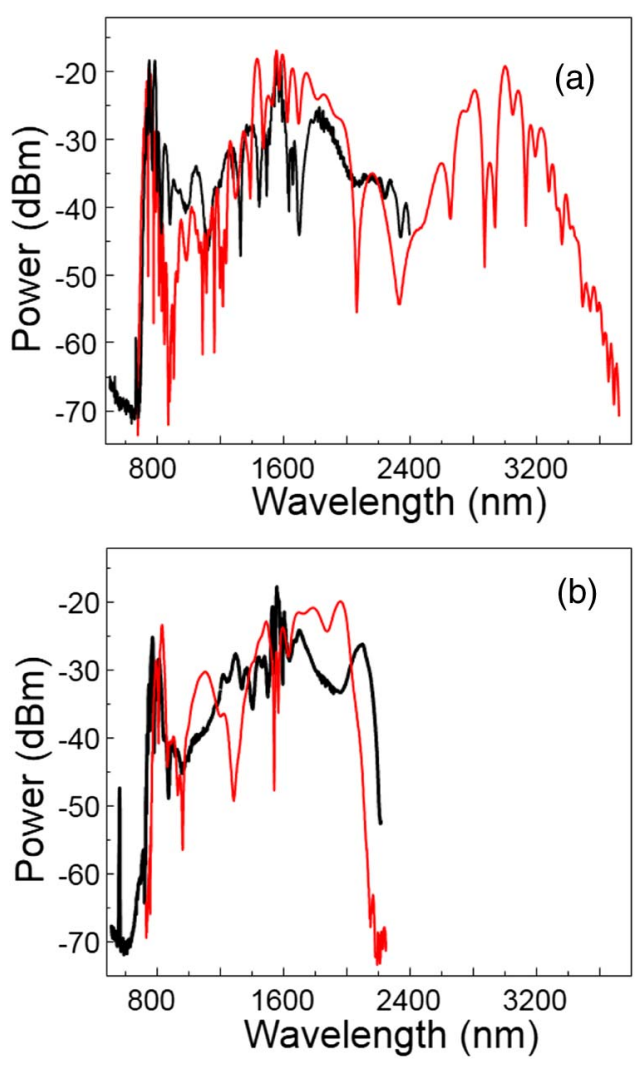

Fig. 13. Measured SC spectrum (black line). Simulated SC spectrum using the measured dispersion profile (red line). (a) Quasi-TE mode and (b) quasi-TM mode.

a slightly narrower bandwidth. For the quasi-TE mode there is almost perfect agreement both for the location of the DW1 and the general shape of SC. Note that in the simulation, the DW2 is located at $3000 \mathrm{~nm}$ and therefore the SC extends from 710 to $3400 \mathrm{~nm}$. Even though the OSA stops measuring at $2400 \mathrm{~nm}$, the simulation indicates that very large bandwidths can be reached with flattened dispersion profiles that can be obtained with multicladding $\mathrm{Si}_{X} \mathrm{~N}_{Y}$ waveguides but not with single-cladding ones. Therefore, the SC bandwidth in our case outperforms other results, which come from single-clad integrated waveguides $[11,14,16,35-39]$.

An analysis of the disagreement between the chromatic dispersion measurements and simulations in Figs. 5(a), 7(a), 8(a), 10(a), and 12 shows that a common trend is that the simulation with the nominal dimensions produces a dispersion profile that is vertically downshifted [Figs. 5(a), 7(a), and 8(a)] or shifted to longer wavelengths [Figs. 5(a), 7(a), 10(a), and 12] with respect to the measurement. Furthermore, the locations of the dispersive waves in the SC simulations obtained using the measured dispersion are in quite good agreement with the measurements, though in general the measured DWs are located at shorter wavelengths [Figs. 6(a), 7(b), 10(b), and 13(b)]. This confirms that the real dispersion is indeed shifted to shorter wavelengths if compared to the simulation using the nominal dimensions. This disagreement cannot be explained as due only to dimension fluctuations but should have an important refractive index contribution. These results might reflect the limitations of the PECVD process to deliver a reproducible refractive index value. 

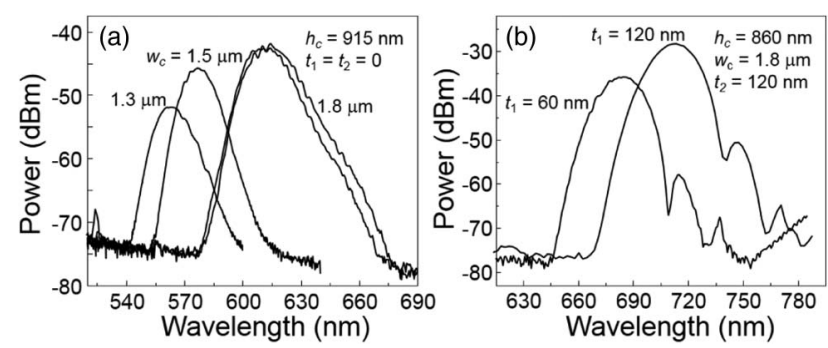

Fig. 14. Tuning of the generated dispersive wave (a) single-cladding waveguide, changing $w_{c}$, and (b) multicladding waveguide, changing $t_{1}$.
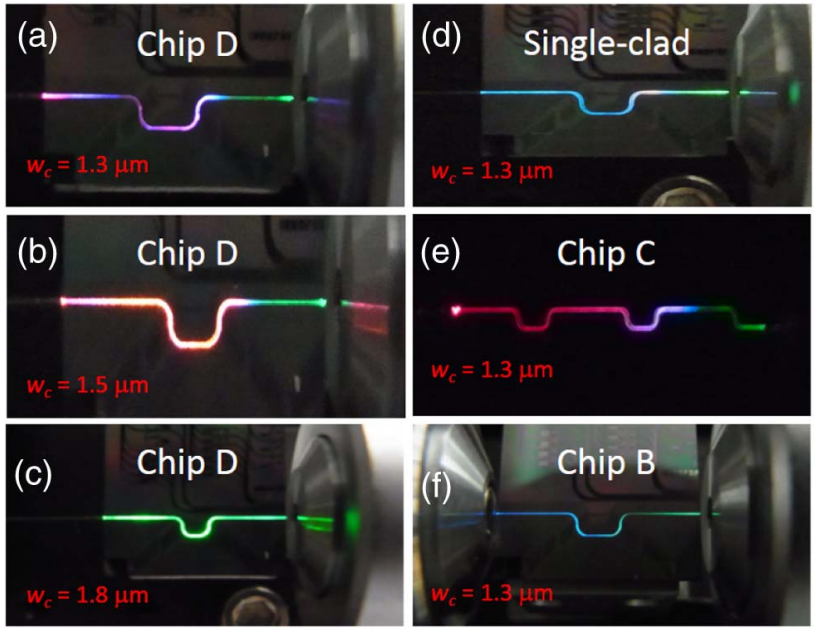

Fig. 15. Pictures of the third harmonic scattered light. Table 2 gives the details of each waveguide. Light comes from right to left.

\section{Dispersive Wave Generation}

Dispersive wave generation has emerged as a wavelength conversion technique to achieve isolated spectral bands in the visible or the infrared wavelength range. Therefore, the ability to engineer the $\mathrm{Si}_{X} \mathrm{~N}_{Y}$ waveguide dispersion can allow tuning of the dispersive wave bands over broad spectral ranges. Figure 14 shows two examples of how the change of a single parameter of the waveguide allowed us to tune the dispersive wave spectrum. Figure 14(a) shows the spectra for three $\mathrm{Si}_{X} \mathrm{~N}_{Y}$ single-cladding waveguides with $h_{c}=915 \mathrm{~nm}$ and $w_{c}=1.3,1.5$, and $1.8 \mu \mathrm{m}$. The dispersive wave central wavelengths are shifted from $562 \mathrm{~nm}$ to $577 \mathrm{~nm}$ and $610 \mathrm{~nm}$. For the dispersive wave centered at $610 \mathrm{~nm}$, two spectra are plotted corresponding to two different waveguides in a chip with identical dimensions. This shows that the dimension and refractive index fluctuations among contiguous waveguides during fabrication are negligible. Figure 14(b) shows the effect of changing $t_{1}$ in the multicladding waveguides. The increase of $t_{1}$ from 60 to $120 \mathrm{~nm}$ produces a spectral shift of the dispersive wave location from 685 to $715 \mathrm{~nm}$.

\section{Third Harmonic Generation (THG)}

Figure 15 offers a series of pictures that show the scattered light from $\mathrm{Si}_{X} \mathrm{~N}_{Y}$ waveguides as they are excited with the femtosecond laser. This excitation generated several colors from blue to red at different propagation sections of the waveguide. Table 2 describes the parameters of the waveguides used to generate the light shown in these pictures and which mode was excited. For each set
Table 2. Parameters of $\mathrm{Si}_{X} \mathrm{~N}_{Y}$ Waveguides for $\mathrm{THG}^{a}$

\begin{tabular}{ccccccc}
\hline Figure & $\begin{array}{c}h_{c} \\
(\mathrm{~nm})\end{array}$ & $\begin{array}{c}w_{c} \\
(\mu \mathrm{m})\end{array}$ & $\begin{array}{c}t_{1} \\
(\mathrm{~nm})\end{array}$ & $\begin{array}{c}t_{2} \\
(\mathrm{~nm})\end{array}$ & Mode & $\begin{array}{c}\text { Length } \\
(\mathrm{cm})\end{array}$ \\
\hline a) & 750 & 1.3 & 40 & 200 & $\mathrm{TE}$ & 1.4 \\
$\mathrm{~b})$ & 750 & 1.5 & 40 & 200 & $\mathrm{TE}$ & 1.4 \\
$\mathrm{c})$ & 750 & 1.8 & 40 & 200 & $\mathrm{TE}$ & 1.4 \\
$\mathrm{~d})$ & 775 & 1.3 & 0 & 0 & $\mathrm{TE}$ & 1.4 \\
$\mathrm{e})$ & 860 & 1.3 & 120 & 120 & $\mathrm{TE}$ & 2.9 \\
$\mathrm{f})$ & 860 & 1.3 & 90 & 120 & $\mathrm{TM}$ & 1.4 \\
\hline
\end{tabular}

${ }^{a}$ All multicladding waveguides have $\mathrm{Si}_{X} \mathrm{~N}_{Y} / \mathrm{SiO}_{2}$ refractive index contrast.
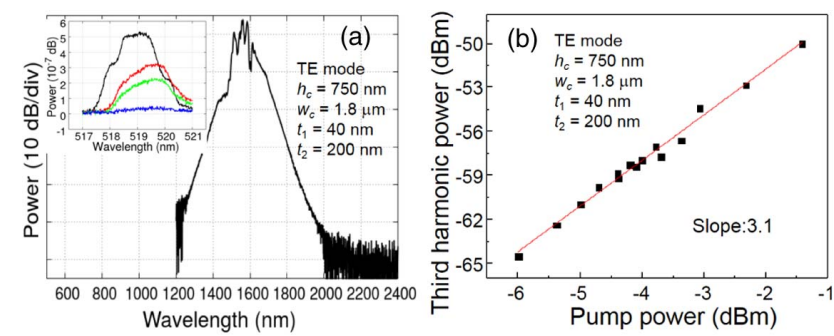

Fig. 16. (a) SC spectrum after $2.5 \mathrm{~mm}$ propagation. Inset: spectrum of the THG as a function of the pump power. (b) Third harmonic power as a function of pump power.

of waveguide parameters, a particular sequence of colors was produced. Figures 15(a)-15(c), for example, show that just varying the core width drastically altered the nonlinear dynamics. However, one common fact of those pictures is that the green light is generated first and that this happens immediately after the femtosecond pulse enters into the $\mathrm{Si}_{X} \mathrm{~N}_{Y}$ waveguide. Then, as the femtosecond pump propagates, the scattered light changes its color [except in Fig. 15(c), where the scattered light remains as a very intense green signature].

To better understand the origin of this intense light, chip $D$ [Figs. 15(a)-15(c)] was cut to make waveguides of $2.5 \mathrm{~mm}$ length. The waveguide for our analysis is the one with nominal parameters of $h_{c}=750 \mathrm{~nm}, w_{c}=1.8 \mu \mathrm{m}, t_{1}=40 \mathrm{~nm}$, and $t_{2}=200 \mathrm{~nm}$. We excited the quasi-TE mode of this waveguide with $P_{0} \approx 500 \mathrm{~W}$ peak power of the femtosecond pump. Figure 16(a) shows the resulting SC spectrum, which has a bandwidth that is much smaller than the one in Fig. 13(a). For this $2.5 \mathrm{~mm}$ long waveguide, only a single peak was generated at $519 \mathrm{~nm}$, which is shown in the inset of Fig. 16(a) for several pump powers. We plotted the measured power of the light at $519 \mathrm{~nm}$ as a function of the input pump power in Fig. 16(b) using a logarithmic scale. By fitting the experimental data with a linear function, a slope of 3.1 is found, indicating that this light originated from a third harmonic process.

Figure 17 shows a comparison of the quasi-TE and quasi-TM third harmonic spectra in the same waveguide of Fig. 16 as generated after a propagation length of $L=2.5 \mathrm{~mm}$ [Fig. 17(a)] and $L=1.4 \mathrm{~cm}$ [Fig. 17(b)], respectively. For the very short waveguide, the generated spectrum is centered at $\approx 519 \mathrm{~nm}$. However, while the linewidth is very narrow for the quasi-TE mode, the third harmonic light for the quasi-TM case is significantly broader. As the femtosecond laser propagates through the waveguide, multiple peaks with intensities that differ by more than one order of magnitude are generated, as shown in Fig. 17(b). To visualize the shortest wavelength for THG, we obtained Fig. 17(b) using 

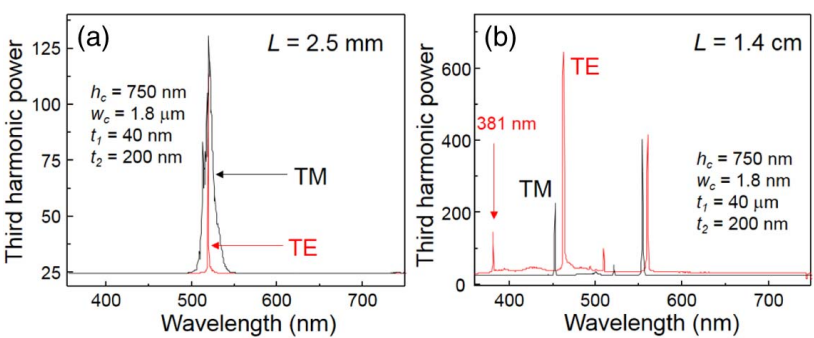

Fig. 17. Third harmonic spectrum for quasi-TE and quasi-TM propagation. (a) After $2.5 \mathrm{~mm}$ and (b) after $1.4 \mathrm{~cm}$.

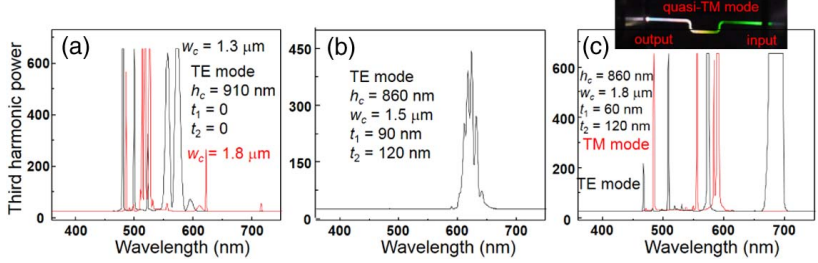

Fig. 18. THG for (a) single-cladding waveguide (as a function of $w_{c}$ ), (b) multicladding waveguide, and (c) multicladding waveguide (as a function of the propagation mode).

optical bandpass filters inserted to select the spectral band from 350 to $500 \mathrm{~nm}$. Note that the shortest measured wavelength is in the ultraviolet at $381 \mathrm{~nm}$. The third harmonic light generated at the very beginning of the waveguide is located at $519 \mathrm{~nm}$ because at the input only the femtosecond pump (centered at $1560 \mathrm{~nm}$ with a bandwidth of $80 \mathrm{~nm}$ ) is present. As the pump propagates and its spectrum is broadened through SC generation, the new noise SC components generate additional THG components.

Figure 18 shows the diversity of THG. The spectrum for the $\mathrm{Si}_{X} \mathrm{~N}_{Y}$ waveguide with $h_{c}=915 \mathrm{~nm}, t_{1}=t_{2}=0 \mathrm{~nm}$ is depicted in Figure 18(a), where we compare two widths: $w_{c}=1.3 \mu \mathrm{m}$ and $1.8 \mu \mathrm{m}$. We excited the quasi-TE mode and did not use optical filtering in this measurement. The multiple peaks due to THG range between 470 to $715 \mathrm{~nm}$. On the other hand, Fig. 18(b) shows the spectrum for a $\mathrm{Si}_{X} \mathrm{~N}_{Y}$ waveguide from chip $B$, where all peaks are packed in the spectral region from 600 to $650 \mathrm{~nm}$. Figure 18(c) shows the THG spectra for chip $A\left(w_{c}=1.8 \mu \mathrm{m}\right)$ plotted for the quasi-TE and quasi-TM propagation. The inset of Fig. 18(c) shows a picture of the scattered light for the quasi-TM mode. The initial intense green light changed its color after less than one $\mathrm{cm}$ and becomes yellow, then white/red, and finally some blue was noted. This result was in agreement with the measured spectrum that exhibited peaks at $485 \mathrm{~nm}$, at $555 \mathrm{~nm}$ and two close to $600 \mathrm{~nm}$.

We calculated the effective refractive index for the first 25 propagating quasi-TE modes in the spectral interval from 380 to $650 \mathrm{~nm}$. The black lines in Fig. 19(a) show the results for the waveguide with $w_{c}=1.8 \mu \mathrm{m}$ from chip $D$. We plotted the effective refractive index of the fundamental mode with a blue line from 1150 to $1950 \mathrm{~nm}$. The many intersection points of the fundamental mode with high-order modes show the wavelengths where there is phase matching for THG. This explains the multiple peaks that appear at the output of the waveguide. The inset of Fig. 19(a) shows a picture of the filtered third harmonic light captured using a piece of paper placed after the filter. The non-Gaussian profile of this green THG light is readily noted. Since the SC spectral broadening happens as the pump propagates, the several THG peaks might have their maximum intensity for different waveguide
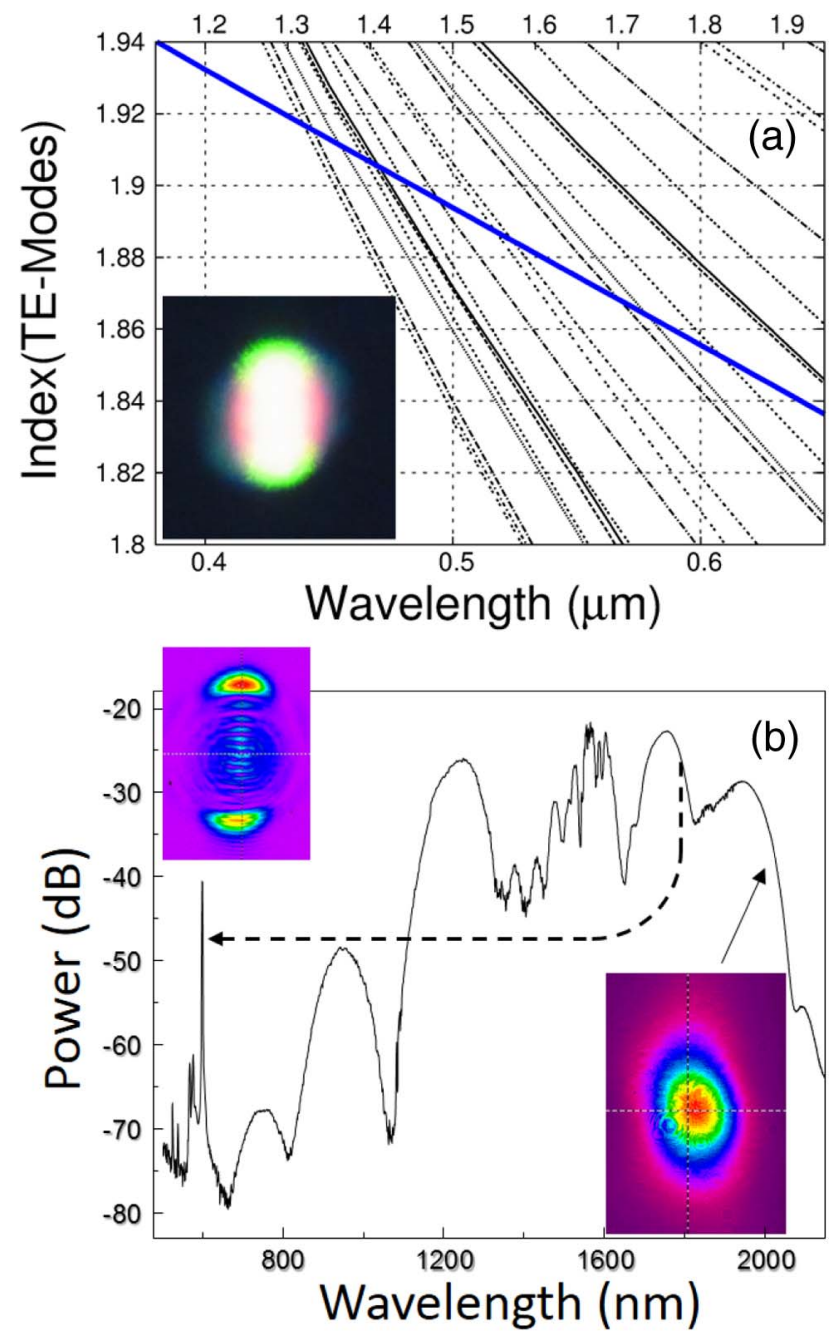

Fig. 19. (a) Effective refractive index for fundamental (blue) and highorder (black lines) propagation modes. (b) SC together with THG tones. The dashed arrow shows the efficiency of THG at $597.5 \mathrm{~nm}$. Transverse profile of THG is shown with the inset on the left. For the SC light it is shown with the inset on the right.

lengths.

The efficiency of THG can be indirectly evaluated from Fig. 19(b). We generated the intense peak at $597.5 \mathrm{~nm}$ (power level of $-40.5 \mathrm{~dB}$ ) from SC spectral components at $1792.5 \mathrm{~nm}$ (power level of -26.4 $\mathrm{dB})$, as shown by the dashed arrow. The relative intensity between them is $-14.1 \mathrm{~dB}$, which indicates a large THG efficiency. While the exact conversion efficiency remains to be verified by measuring the THG power with a narrow linewidth laser at $1792.5 \mathrm{~nm}$, in our experiments we measured $\sim 100 \mathrm{nW}$ of out-coupled power at $597.5 \mathrm{~nm}$, which means that $0.5 \mu \mathrm{W}$ average power was generated inside the waveguide. This result indicates a larger efficiency in our $\mathrm{Si}_{X} \mathrm{~N}_{Y}$ waveguides when compared with results reported in $[13,34,35]$. In our waveguides, the largest efficiency occurred between 560 and $600 \mathrm{~nm}$, although the reason for this is still unclear. We measured the spatial profile of THG after filtering out the SC light. The inset on the left shows the transverse profile that has multiple maxima and minima, a signature of high-order mode propagation. On the other hand, the Gaussian transverse profile of the SC light (inset on the right) shows that the pump propagates in the fundamental mode. 

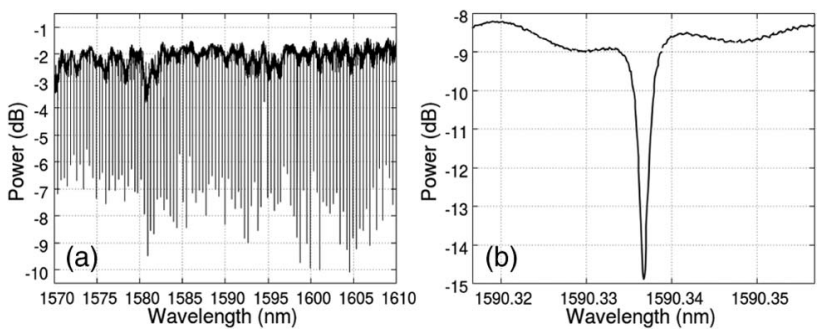

Fig. 20. (a) Transmission spectrum after scanning a laser from 1570 to $1610 \mathrm{~nm}$ and (b) zoom of resonance at $1590.3 \mathrm{~nm}$.

\section{Conclusions}

We investigated multicladding silicon nitride waveguides with engineered effective refractive index for efficient supercontinuum and THG. To assess the performance of the engineering procedure, we compared measurements and simulations of the chromatic dispersion that were complemented with an experiment/simulation analysis of the wavelength location of generated dispersive waves. Both dimension and refractive index fluctuations in our PECVD process contributed to a dispersion unpredictability of $\sim 20 \mathrm{ps} / \mathrm{nm} / \mathrm{km}$.

Contrary to a low-pressure CVD process that results in reproducible and pure $\mathrm{Si}_{X} \mathrm{~N}_{Y}$ waveguides, the PECVD process seems to lack the reproducibility necessary for dispersion engineering with $\mathrm{ps} / \mathrm{nm} / \mathrm{km}$ precision. Nevertheless, we demonstrated multicladding waveguides exhibiting low $(<35 \mathrm{ps} / \mathrm{nm} / \mathrm{km})$ and anomalous dispersion over a $900 \mathrm{~nm}$ bandwidth, which single-cladding waveguides cannot offer. Refining the fabrication process might offer better control of the refractive index and dimensions of the waveguide on even broader bandwidths of nonlinear conversion than we reported here (from ultraviolet to $\sim 3.3 \mu \mathrm{m}$ ).

\section{Appendix A}

An accurate value of the propagation loss in $\mathrm{Si}_{X} \mathrm{~N}_{Y}$ waveguides comes from $Q$-factor measurements in ring resonators, which are fabricated on the same chip containing the waveguides where we performed the nonlinear experiments. We characterized the resonances through the wavelength scanning of a laser finely tuned from 1520 to $1610 \mathrm{~nm}$. Figure 20(a) shows the resonances of a ring resonator with $250 \mu \mathrm{m}$ radius and coming from the same chip as the one used in Figs. 5 and 6. We restricted the plot to 1570$1610 \mathrm{~nm}$, where the H-N losses are smaller. Figure 20(b) shows the transmission spectrum centered at one of the resonances. By measuring the resonance width, $\Delta f$, the $Q$-factor is obtained using $Q=f / \Delta f$. We found that the measured $Q$-factor in this spectral region has an average value of $0.75 \times 10^{6}$, which corresponds to a material loss of $0.35 \mathrm{~dB} / \mathrm{cm}$. The same measurement was performed in other ring resonators from the same chip and the average $Q$-factor was found to fluctuate between 0.35 and $1.05 \times 10^{6}$.

We did the loss characterization of the other chips through a simple comparison with the chip in Fig. 20. Light is injected into the waveguides of the chip from Fig. 20 and also into the other chips and the difference in the propagation loss is measured. We estimate that most of the multicladding $\mathrm{Si}_{X} \mathrm{~N}_{Y}$ waveguides have losses ranging from 0.5 to $1 \mathrm{~dB} / \mathrm{cm}$.

\section{Funding}

Bundesministerium für Bildung und Forschung (BMBF) (03Z2AN11, 03Z2AN12).

\section{Acknowledgment}

We gratefully acknowledge V. Torres Company and A. Fülöp from Chalmers University of Technology for the $Q$-factor measurements.

\section{References}

1. P. St. J. Russell, "Photonic crystal fibers," Science 299, 358-362 (2003).

2. K. P. Hansen, "Dispersion flattened hybrid-core nonlinear photonic crystal fiber," Opt. Express 11, 1503-1509 (2003).

3. Th. Udem, R. Holzwarth, and T. W. Hänsch, "Optical frequency metrology," Nature 416, 233-237 (2002).

4. T. A. Birks, W. J. Wadsworth, and P. St. J. Russell, "Supercontinuum generation in tapered fibers," Opt. Lett. 25, 1415-1417 (2000).

5. B. Jalali, "Teaching silicon new tricks," Nat. Photonics 1, 193-195 (2007).

6. J. Leuthold, C. Koos, and W. Freude, "Nonlinear silicon photonics," Nat. Photonics 4, 535-544 (2010).

7. H. Rong, A. Liu, R. Jones, O. Cohen, D. Hak, R. Nicolaescu, A. Fang, and M. Paniccia, "A continuous-wave Raman silicon laser," Nature 433, 725-728 (2005)

8. M. A. Foster, A. C. Turner, J. E. Sharping, B. S. Schmidt, M. Lipson, and A. L. Gaeta, "Broad-band optical parametric gain on a silicon photonic chip," Nature 441, 960-963 (2006).

9. B. Kuyken, X. Liu, G. Roelkens, R. Baets, R. M. Osgood, and W. M. J. Green, " $50 \mathrm{~dB}$ parametric on-chip gain in silicon photonic wire," Opt. Lett. 36, 4401-4403 (2011).

10. F. Ferdous, H. Miao, D. E. Leaird, K. Srinivasan, J. Wang, L. Chen, L. T. Varghese, and A. M. Weiner, "Spectral line-by-line pulse shaping of on-chip microresonator frequency combs," Nat. Photonics 5, 770-776 (2011).

11. D. Duchesne, M. Peccianti, M. R. E. Lamont, M. Ferrera, L. Razzari, F. Lgar, R Morandotti, S. Chu, B. E. Little, and D. J. Moss, "Supercontinuum generation in a high index doped silica glass spiral waveguide," Opt. Express 18, 923-930 (2010).

12. Y. Okawachi, K. Saha, J. S. Levy, Y. H. Wen, M. Lipson, and A. L. Gaeta, "Octave-spanning frequency comb generation in a silicon nitride chip," Opt. Lett. 36, 3398-3400 (2011).

13. J. S. Levy, M. A. Foster, A. L. Gaeta, and M. Lipson, "Harmonic generation in silicon nitride ring resonators," Opt. Express 19, 415-421 (2011).

14. R. Halir, Y. Okawachi, J. S. Levy, M. A. Foster, M. Lipson, and A. L. Gaeta, "Ultrabroadband supercontinuum generation in a CMOS-compatible platform," Opt. Lett. 37, 1685-1687 (2012).

15. T. Herr, K. Hartinger, J. Riemensberger, C. Y. Wang, E. Gavartin, R. Holzwarth, M. L. Gorodetsky, and T. J. Kippenberg, "Universal formation dynamics and noise of Kerr-frequency combs in microresonators," Nat. Photonics 6, 480-487 (2012).

16. F. Leo, J. Safioui, B. Kuyken, G. Roelkens, and S.-P. Gorza, "Generation of coherent supercontinuum in a-Si:H waveguides: experiment and modeling based on measured dispersion profile," Opt. Express 22, 28997-29007 (2014).

17. H. Jung, C. Xiong, K. Y. Fong, X. Zhang, and H. X. Tang, "Optical frequency comb generation from aluminum nitride microring resonator," Opt. Lett. 38, 2810-2813 (2013).

18. E. Dulkeith, F. Xia, L. Schares, W. M. Green, and Y. A. Vlasov, "Group index and group velocity dispersion in silicon on-insulator photonic wires," Opt Express 14, 3853-3863 (2006).

19. X. Liu, W. M. Green, I.-W. Hsieh, J. I. Dadap, Y. A. Vlasov, and R. M Osgood, "Conformal dielectric overlayers for engineering dispersion and effective nonlinearity of silicon nanophotonic wires," Opt. Lett. 33, 2889-2891 (2008).

20. L. Zhang, Y. Yue, R. G. Beausoleil, and A. E. Willner, "Flattened dispersion in silicon slot waveguides," Opt. Express 18, 20529-20534 (2010).

21. D. T. H. Tan, K. Ikeda, P. C. Sun, and Y. Fainman, "Group velocity dispersion and self-phase modulation in silicon nitride waveguides," Appl. Phys. Lett. 96, 061101 (2010).

22. L. Zhang, Q. Lin, Y. Yue, Y. Yan, R. G. Beausoleil, and A. E. Willner, "Silicon waveguide with four zero-dispersion wavelengths and its application in on-chip octave-spanning supercontinuum generation," Opt. Express 20, 1685-1690 (2012).

23. Y. Yue, L. Zhang, H. Huang, R. G. Beausoleil, and A. E. Willner, "Silicon-onnitride waveguide with ultralow dispersion over an octave-spanning mid-infrared wavelength range," IEEE Photonics J. 4, 126-132 (2012).

24. J. Riemensberger, K. Hartinger, T. Herr, V. Brasch, R. Holzwarth, and T. J. Kippenberg, "Dispersion engineered high-Q silicon nitride ring-resonators via atomic layer deposition," Opt. Express 20, 27661-27669 (2012).

25. F. Leo, U. Dave, S. Keyvaninia, B. Kuyken, and G. Roelkens, "Measurement and tuning of the chromatic dispersion of a silicon photonic wire around the half band gap spectral region,” Opt. Lett. 39, 711-714 (2014). 
26. C. Bao, Y. Yan, L. Zhang, Y. Yue, N. Ahmed, A. M. Agarwal, L. C. Kimerling, J. Michel, and A. E. Willner, "Increased bandwidth with flattened and low dispersion in a horizontal double-slot silicon waveguide," J. Opt. Soc. Am. B 32, 26-30 (2015).

27. J. M. Chavez Boggio, D. Bodenmüller, T. Fremberg, R. Haynes, M. M. Roth, R. Eisermann, M. Lisker, L. Zimmermann, and M. Böhm, "Dispersion engineered silicon nitride waveguides by geometrical and refractive-index optimization," J. Opt. Soc. Am. B 31, 2846-2857 (2014).

28. N. Akhmediev and M. Karlsson, "Cherenkov radiation emitted by solitons in optical fibers," Phys. Rev. A 51, 2602-2607 (1995).

29. A. V. Husakou and J. Herrmann, "Supercontinuum generation of higher-order solitons by fission in photonic crystal fibers," Phys. Rev. Lett. 87, 203901 (2001).

30. I. Cristiani, R. Tediosi, L. Tartara, and V. Degiorgi, "Dispersive wave generation by solitons in microstructured optical fibers," Opt. Express 12, 124-135 (2004).

31. J. M. Dudley, G. Genty, and S. Coen, "Supercontinuum generation in photonic crystal fiber," Rev. Mod. Phys. 78, 1135-1184 (2006).

32. D. Modotto, M. Andreana, K. Krupa, G. Manili, U. Minoni, A. Tonello, V. Couderc, A. Barthélémy, A. Labruyère, B. M. Shalaby, P. Leproux, S. Wabnitz, and A. B. Aceves, "Efficiency of dispersive wave generation in dual concentric core microstructured fiber," J. Opt. Soc. Am. B 32, 1676-1685 (2015).

33. T. Carmon and K. J. Vahala, "Visible continuous emission from a silicon microphotonic device by third-harmonic generation," Nat. Phys. 3, 430-435 (2007).

34. T. Ning, O. Hyvrinen, H. Pietarinen, T. Kaplas, M. Kauranen, and G. Genty, "Third-harmonic UV generation in silicon nitride nanostructures," Opt. Express 21, 2012-2017 (2013).

35. J. P. Epping, T. Hellwig, M. Hoekman, R. Mateman, A. Leinse, R. G. Heideman, A. van Rees, P. J. M. van der Slot, C. J. Lee, C. Fallnich, and K.-J. Boller, "Simultaneous supercontinuum and third harmonic generation in $\mathrm{Si}_{3} \mathrm{~N}_{4}$ waveguides," in European Conference on Lasers and Electro-Optics (ECLE) (2015), paper CK_5_.

36. H. Zhao, B. Kuyken, S. Clemmen, F. Leo, A. Subramanian, A. Dhakal, P. Helin, S. Severi, E. Brainis, G. Roelkens, and R. Baets, "Visible-to-near-infrared octave spanning supercontinuum generation in a silicon nitride waveguide," Opt. Lett. 40, 2177-2180 (2015).

37. J. P. Epping, T. Hellwig, M. Hoekman, R. Mateman, A. Leinse, R. G. Heideman, A. van Rees, P. J. M. van der Slot, C. J. Lee, C. Fallnich, and K.-J. Boller, "On-chip visible-to-infrared supercontinuum generation with more than 495 THz spectral bandwidth,” Opt. Express 23, 19596-19604 (2015).

38. F. Leo, S. P. Gorza, S. Coen, B. Kuyken, and G. Roelkens, "Coherent supercontinuum generation in a silicon photonic wire in the telecommunication wavelength range," Opt. Lett. 39, 711-714 (2014).

39. X. Liu, M. Pu, B. Zhou, C. J. Krückel, A. Fülöp, V. Torres-Company, and M. Bache, "Octave-spanning supercontinuum generation in a silicon-rich nitride waveguide," Opt. Lett. 41, 2719-2722 (2016). 